We also analyzed the characteristics of super-spreaders, factors associated with mortality, and hospital response to infection. Results: The first mortality by MERS-CoV in Korea was infected by the first super-spreader in Korea. The lessons after the outbreak were as follows: - A higher index of alerting system to find the source-patient earlier. - Appropriate numbers of Airborne Infection Isolation Rooms (AIIRs) should be constructed and maintained. - Proper training on putting on and take off of Personal Protective Equipment. - Well-trained health care workers to care for patients infected with highly contagious pathogens must be fostered. - Crowded and narrow hospital rooms should be converted to visitor controlled, larger-spaced hospital rooms.

Conclusion: Multiple potential factors were associated with the super-spreading events: misdiagnosis, delayed hospital admission, inter-hospital transfers without accurate information, and also behaviors such as ignoring instructions regarding infection control, and poor environmental conditions. Institutional and health care systems' preparedness is required to prevent such outbreaks.

Prehosp Disaster Med 2017;32(Suppl. 1):s4-s5

doi:10.1017/S1049023X17000401

Training and Preparedness for CBRN Emergencies in a Conflict Zone, Lebanon

Nagi Souaiby

Faculty Of Medicine, St Joseph University, Beirut/Lebanon

Study/Objective: Providing training and preparedness for Chemical, Biological, Radiological and Nuclear (CBRN) emergencies to local actors, will increase knowledge and skills of the disaster response community and health care providers, and prepare them for undertaking future responses, while providing training to both local and international actors, will increase the response capacity of humanitarian relief workers who have a large presence in border areas of and among Syrian refugee populations.

Background: Following the chemical attack in Syria, with the resulting mass casualties, Lebanese Ministry of Health, with the support of the World Health Organization and in cooperation with the Lebanese Syndicate of Hospitals, worked on increasing the preparedness and response capabilities of healthcare providers, especially those situated near Syrian borders. Concerned parties and responsible stakeholders became more interested and aware of the importance of training field workers on CBRN emergencies.

Methods: Eleven workshops were offered throughout Lebanon (North, Beirut, Bekaa, South); 8 of which were dedicated to non clinical staff (total of 207) and 3 to clinical ones (total of 105). It was facilitated using multiple methods to engage participants and reinforce messages. It was delivered in English and/or Arabic. Tools included videos, PowerPoint presentations, case studies and group exercises.

Results: The pre/post tests allowed for evaluating trainees; the evolution percentage for the Non clinical staff ranged from a minimum of $19 \%$ (Beirut) to a maximum of $49 \%$ (Tyr). As for clinical staff, it ranged from $8 \%$ (Tripoli 3) to $45 \%$ (Beirut 3).
Conclusion: Following the international community and the Non-Governmental Organizations (NGOs) effort and urgent need, the CBRN National Team in Lebanon was founded. It is headed by the Secretary General of the Higher Council of Defense and composed of representatives from all relevant parties. CBRN incidents present various challenges at all levels, including decision makers and first responders. Continuous training and preparations with strong cooperation and coordination between all parties, may decrease the impact of such event. A lot remains to be done in this regard where further research is needed.

Prehosp Disaster Med 2017;32(Suppl. 1):s5 doi:10.1017/S1049023X17000413

\section{Live Animal CBRN Surveillance: The XIV Pan-American Games Case Experience \\ Amado A. Baez}

Emergency Medicine, Jackson Memorial Hospital, Miami/FL/United States of America

Study/Objective: Develop and implement a comprehensive live animal Chemical Biological Radiological and Nuclear (CBRN) surveillance program to support the XIV Pan-American Games.

Background: After the September 11, 2001 terrorist attacks, preparedness and response was raised at international sports events, including enhanced surveillance and rapid detection of terrorist-induced or natural events for a timely intervention. The Pan-American Games are the fourth most important international athletic event in the world. Hosted by the city of Santo Domingo, DR, the XIV Pan-American Games Security Directorate developed a CBRN unit.

Methods: The unit had strategic and operational mandates. For operational support, two strike teams were active at any given time, each team consisted of five members including a team leader, field physician, and tactical officers. Syndrome surveillance was performed by means of direct communications between the hospitals and units, as well as use of an electronic Web-based surveillance tool. For active real-time surveillance and recognizing the value of the lethal dose 50 concept ( LD50 is the dose of a substance required to kill half the members of a tested population, the LD50 is body mass dependant), a live animal surveillance station (LASS) program was developed and placed in strategic areas. The LASS consisted of bird cages located in confined spaces and a fresh water fish tank with a continuous stream.

Results: Bird stations were placed at VIP areas at major sporting venues and a small fish tank emanating from the centralized water tank supplying the Pan-American village, all monitored $24 / 7$ by webcams. Early morning the day of the opening of the village, the surveillance system identified dead fish in the tank. Investigations found non-malicious cause related to over chlorination of the water pipe system; this incident prompted activation and testing of the emergency response protocol.

Conclusion: Live animal stations offers a cost-effective surveillance method for CBRN support units.

Prehosp Disaster Med 2017;32(Suppl. 1):s5

doi:10.1017/S1049023X17000425 